\title{
Measuring Inaccessible Residual Stresses Using Multiple Methods and Superposition
}

\author{
P. Pagliaro • M.B. Prime • J.S. Robinson • B. Clausen • \\ H. Swenson • M. Steinzig $•$ B. Zuccarello
}

Received: 22 April 2010 / Accepted: 10 October 2010 /Published online: 20 November 2010

(C) The Author(s) 2010. This article is published with open access at Springerlink.com

\begin{abstract}
The traditional contour method maps a single component of residual stress by cutting a body carefully in two and measuring the contour of the cut surface. The cut also exposes previously inaccessible regions of the body to residual stress measurement using a variety of other techniques, but the stresses have been changed by the relaxation after cutting. In this paper, it is shown that superposition of stresses measured post-cutting with results from the contour method analysis can determine the original (pre-cut) residual stresses. The general superposition theory using Bueckner's principle is developed and limitations are discussed. The procedure is experimentally demonstrated by determining the triaxial residual stress state on a cross section plane. The 2024T351 aluminum alloy test specimen was a disk plastically indented to produce multiaxial residual stresses. After cutting the disk in half, the stresses on the cut surface of one half were determined with X-ray diffraction and with hole drilling on the other half. To determine the original residual stresses, the measured surface stresses were superimposed with the change stress calculated by the contour method. Within uncertainty, the results agreed with neutron diffraction measurements taken on an uncut disk.
\end{abstract}

P. Pagliaro: Visiting student at Los Alamos National Laboratory.

P. Pagliaro · B. Zuccarello

Dipartimento di Meccanica, Università degli Studi di Palermo, 90128 Palermo, Italy

M.B. Prime $(\bowtie$, SEM member $) \cdot B$. Clausen $\cdot H$. Swenson • M. Steinzig (SEM member)

Los Alamos National Laboratory,

Los Alamos, NM 87545, USA

e-mail: prime@lanl.gov

\section{J. Robinson}

Department of Materials Science and Technology,

University of Limerick,

Limerick, Ireland
Keywords Residual stress · Contour method · Neutron diffraction $\cdot$ Superposition $\cdot$ Sectioning $\cdot$ Hole $\cdot$ Drilling . $\mathrm{X}$-ray diffraction

\section{Introduction}

There are many important applications where it is very difficult to measure internal residual stresses with current technology. One example is axial stresses in girth (circumferential) welds on piping and pressure vessels. Circumferential cracks cause the greatest concern for rupture in nuclear power plants $[1,2]$. To remain in service, such defected components must be demonstrated to be safe against rupture. Axial residual stresses are a main driver for the growth of circumferential cracks and must be known for crack growth and leak-before-break analyses [3-5]. Measurement of those stresses is difficult. Neutron diffraction is the most commonly published technique for measuring internal stresses. However, some components are too thick for neutron measurement and welds are often problematic because of spatial variations in the reference lattice constant caused by chemistry changes in and around the weld [6-8] or the presence of microstresses [8]. The deep hole method [9] has had the most success on very thick components but measures only a 1D stress profile. The contour method [10] can measure a radial-axial cross-section map but only of the hoop stress instead of the axial stress, e.g., [11, 12]. There are many other examples of important applications where no one technique can perform the complete residual stress characterization.

Currently, many larger parts are sectioned or otherwise altered in order to provide access and thereby exploit the capabilities of advanced neutron and synchrotron diffraction instruments [13-23] or laboratory X-rays [24]. Assumptions are often made that the sectioning does not 
change the stresses being measured. Those assumptions should then limit the subsequent measurements to regions of the part unaffected by the sectioning.

This paper presents a procedure for accurately obtaining internal stress access by first sectioning, i.e., cutting, a part in conjunction with a contour method measurement. Then any technique, usually not contour, can be used to measure the partially relaxed stresses at any location, surface or subsurface, that is now accessible in the sectioned part. The same elastic finite element analysis that is used for the traditional contour method data reduction also quantifies the elastic relaxation of all other stresses in the part. Therefore, superposition of the post-sectioning measurements with the appropriate results from the contour method analysis determines the original residual stresses at the measurement locations. The contour method calculations also quantitatively identify regions of the part that are unaffected by sectioning.

The procedure is experimentally validated in this paper for a particular application of the theory: the surface-superposition contour method to measure the triaxial stress state on a plane. After a contour method cut to measure the out-of-plane stress component, the remaining in-plane stresses on the cut surface are measured using two independent techniques: laboratory $\mathrm{X}$-ray diffraction and incremental hole drilling. Superposition of the measured surface stresses with the calculated stress relaxation then determines the original, internal stresses. The results are compared with neutron diffraction measurements.

\section{Principle and Assumptions}

Principle

As a review of previous work [10], Fig. 1 illustrates Bueckner's superposition principle [25] as applied to sectioning parts for residual stress measurement. Step A shows the undisturbed part with the residual stresses that are to be determined. The part is cut in two on the plane $x=$ 0 and deforms as residual stresses are released by the cut. B shows half of the part in the post-cut state with partiallyrelaxed stresses. The surface contour is measured at this point. $\mathbf{C}$ is an analytical step that starts with a stress-free body and then the surface created by the cut is displaced back to its original flat shape. Assuming elasticity, superimposing the partially relaxed stress state in $\mathbf{B}$ with the change in stress from $\mathbf{C}$ gives the original residual stress throughout the part:

$\sigma^{A}(x, y, z)=\sigma^{B}(x, y, z)+\sigma^{C}(x, y, z)$ where $\sigma$ without subscripts refers to the entire stress tensor. $\sigma^{A}$ can be referred to as the original stress, $\sigma^{B}$ as the remaining stress, and $\sigma^{C}$ as the change stress since it quantifies how much the stress has relaxed from sectioning.

Traditional implementation of the contour method only determines the normal stress, $\sigma_{\mathrm{x}}$, and only on the plane of the cut. This paper presents the first use of the superposition principle to measure other stress components and to measure stresses away from the cut plane. To qualitatively illustrate superposition for such stresses, Fig. 2 represents a section taken through $z=0$ in Fig. 1. The figure was made from an elastic, plane strain finite element model with initial stresses used to represent residual stresses. Each half of Fig. 2 was taken from the same finite element analysis, but with different stress components plotted. The left half of the figure is colored by the magnitude of $\sigma_{\mathrm{x}}$ and the right by $\sigma_{\mathrm{z}}$, which was initially equal to $\sigma_{\mathrm{x}}$. Step $\mathbf{B}$ shows that far away from the cut, both stress components are undisturbed from their original value in $\mathbf{A}$. The amount of relaxation increases closer to the cut surface, with $\sigma_{\mathrm{x}}$ fully relaxed on the cut surface as required by the free surface condition. $\sigma_{\mathrm{z}}$ is only partially relaxed on the free surface. The stresses in C from forcing the surface back flat are equal to the stresses that were relaxed by the sectioning.

For experimentally accessing other stress components and stresses away from the cut plane with this superposition
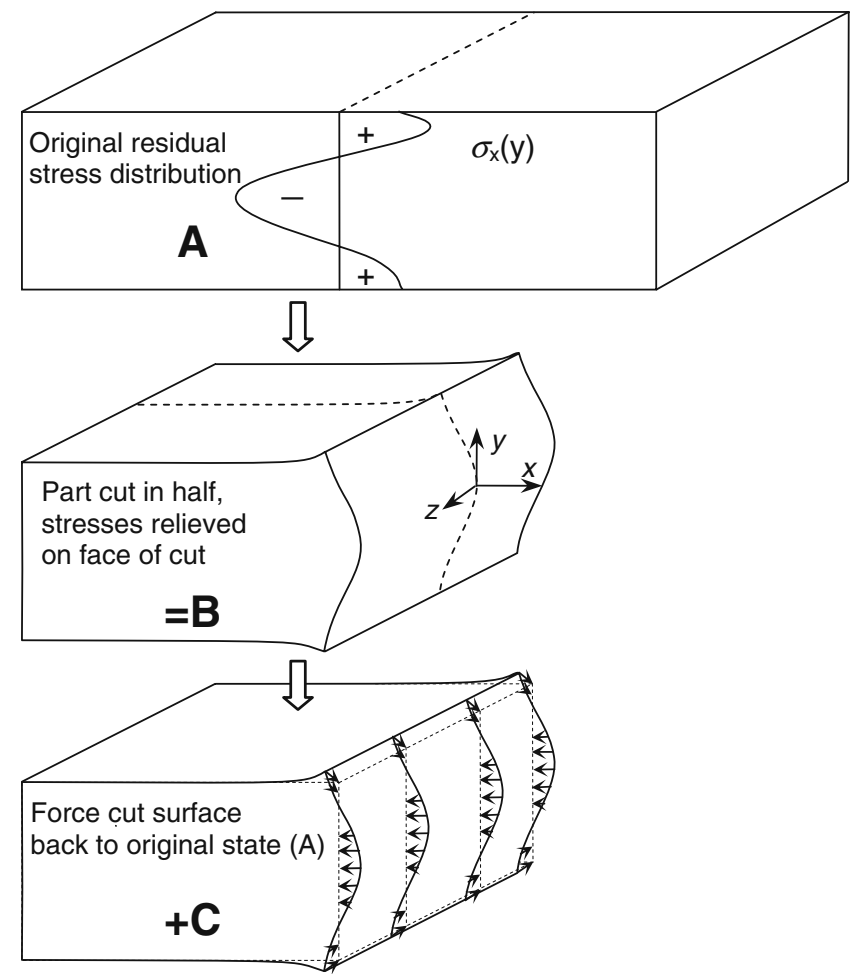

Fig. 1 Bueckner's superposition principle applied to residual stresses in a sectioned part: $\mathbf{A}=\mathbf{B}+\mathbf{C}$ 

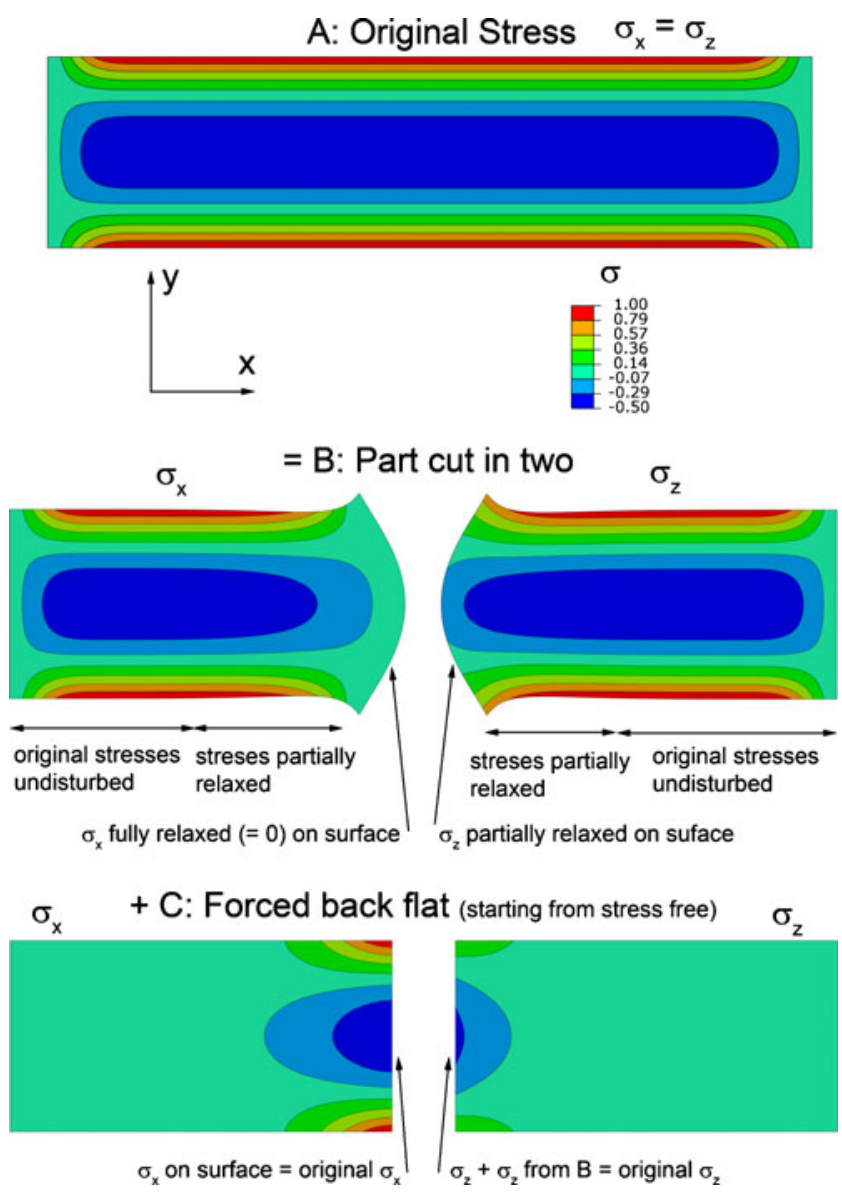

Fig. 2 Two-dimensional plot of elastic superposition principle. Colored by $\sigma_{\mathrm{x}}$ on left half and $\sigma_{\mathrm{z}}$ on right half to show different behaviors of normal stress versus transverse stress. Deformations are exaggerated

principle, the full stress tensor $\sigma^{C}$ is calculated as shown in Fig. 1 from the measured surface contour data with a single, 3-D, elastic finite element (FE) analysis. In the sectioned part, one or more components of the tensor $\sigma^{B}$ are measured at any location using any appropriate technique for residual stress measurement. Simple pointwise superposition with equation (1) returns the original residual stresses, $\sigma^{A}$, for the locations and stress components measured in the sectioned part. Stresses determined by this superposition are called the "reconstructed" stresses.

The contour method calculations also quantitatively identify regions of the part that are unaffected by sectioning. The bottom row in Fig. 2 shows the results of the contour calculations: the change stresses for all stress components throughout the sectioned part. Anywhere that these stresses are of negligible magnitude, the stresses are unaffected by sectioning as can be seen by equation (1) and in Fig. 2. A measurement of the stresses at such locations in the sectioned part gives the original residual stresses without any need for superposition.
It should be emphasized that only a single FE analysis is used for 1) the calculation of the normal stress $\sigma_{\mathrm{x}}$ from the measured surface contour, 2) determining any component of the stress tensor anywhere in the sectioned part by superposition with additional measurements, and 3) for quantification of regions unaffected by the sectioning. The analysis is the one at the bottom of Fig. 1. Displacement conditions are applied to the cut surface to return the material points to their original location. As detailed previously, the simultaneous solution for all of the stresses is unique because it is a Kirchhoff boundary value problem with fully prescribed boundary conditions [10]. The accuracy of the unique solution depends on the validity of the assumptions used to experimentally implement the theory.

\section{Assumptions}

Standard assumptions apply for residual stress measurement using relaxation methods [26]. The stress relaxation after material removal is assumed to occur elastically. Any technique used to section the part is assumed to not introduce significant stresses. It must be assumed that the cut is constant width relative to the undeformed (step $\mathbf{A}$ in Fig. 1) state of the body, a standard contour method assumption about the cut [27]. Satisfying that assumption requires the part to be securely clamped during cutting so that the material at the tip of the cut is not significantly stretched or compressed as cutting progresses and stresses relax.

Additional assumptions unique to the contour method should be discussed considering the superposition approach proposed in this paper. Because the measured contour shape does not provide information about the transverse deformation, displacements in step $\mathbf{C}$ are only applied in the $\mathbf{x}$ direction. Information about the shear stresses released on the plane of the cut, $\tau_{x y}^{A}(0, y, z)$ and $\tau_{x z}^{A}(0, y, z)$ is lost. For the traditional contour method where only the normal stress on the cut surface, $\sigma_{x}^{A}(0, y, z)$, is determined, averaging the contours on the two sides is sufficient to remove errors caused by the released shear stresses [10]. For the general case of measuring other stresses, one must measure the remaining stresses, $\sigma^{B}$, on both halves of the part and average them to ensure accurate results. Numerical simulations indicate, however, that this anti-symmetric shearstress effect is insignificant for most practical applications [28] because those two shear stresses tend to have smaller magnitudes and their effect on $\sigma^{B}$ is reduced relative to normal stresses [29]. Because the contours on the two sides are averaged, it is assumed that the part is geometrically symmetric about the cut plane. Significant stiffness differences on the two sides of the cut, such as cutting very near one end of a long part, can cause errors if not treated correctly. 
Special Case: Surface Superposition

The surface-superposition contour method, a particular implementation of the broader theory, is experimentally validated in this paper. In the surface-superposition contour method, multiple stresses can be determined on the cut surface $x=0$. Equation (1) for this case becomes:

$\sigma_{x}^{A}(0, y, z)=\sigma_{x}^{C}(0, y, z)$

$\sigma_{y}^{A}(0, y, z)=\sigma_{y}^{B}(0, y, z)+\sigma_{y}^{C}(0, y, z)$

$\sigma_{z}^{A}(0, y, z)=\sigma_{z}^{B}(0, y, z)+\sigma_{z}^{C}(0, y, z)$

$\tau_{y z}^{A}(0, y, z)=\tau_{y z}^{B}(0, y, z)+\tau_{y z}^{C}(0, y, z)$.

The first line of equation (2) corresponds to the traditional contour method to measure $\sigma_{\mathrm{x}}$ in which $\sigma_{\mathrm{x}}$ in step B of Figs. 1 and 2 is zero because of the free surface condition. To determine the other stress components by superposition and equation (2), any or all of $\sigma_{y}^{B}, \sigma_{z}^{B}$, and $\tau_{y z}^{B}$ are measured on the cut surface using a technique such as hole drilling or $\mathrm{X}$ ray diffraction.

Because stresses are measured on the cut surface, an additional experimental step and assumption are required. The cutting process generally induces additional stresses in a thin layer on the cut surface [30]. The amount of stressed material is usually too small to have a significant effect on the surface contour measured with the traditional contour method, and so does not invalidate the assumption of stress-free cutting in that case. However, for the surface-superposition contour method, the cutting stresses can have a large effect on surface stresses measured directly on the cut surface. In other words, one wants to measure the surface stresses after elastic relaxation, not the cutting-induced stresses. Therefore, to satisfy the stress-free cutting assumption, the thin layer of material affected by machining must be removed by a stressfree process such as electrochemical layer removal. Considering that the problem is elastic and path independent, removing the layer is equivalent to a cut having been made at the final surface location. It must therefore be assumed that the original residual stresses were not significantly different on the cut plane compared to the new surface location displaced by the thickness of the removed layer, $\delta$ :

$\sigma^{A}(0, y, z) \approx \sigma^{A}(0 \pm \delta, y, z)$

Considering the small thickness of the layer that must be removed, this is usually a very reasonable assumption.

\section{Experimental Validation}

Specimen

A specially designed specimen was used for validation. As described in detail elsewhere and illustrated in Fig. 3, a disk

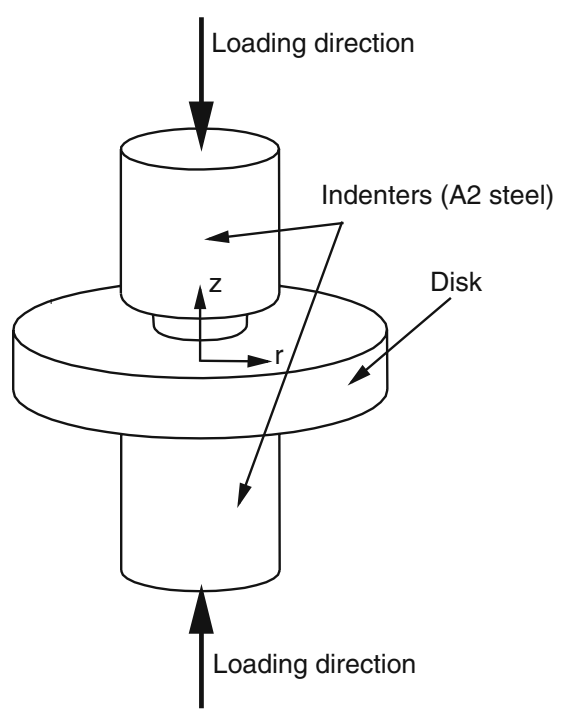

Fig. 3 Schematic of indentation process used to produce residual stresses in disk specimen

was indented to introduce residual stresses from plastic deformation [31-33]. The disk was $60-\mathrm{mm}$ in diameter with a 10-mm thickness chosen to make the part accessible to independent neutron diffraction measurements of residual stresses. Opposing hardened steel indenters loaded 13-mm diameter regions centered on the faces of the disk, and then the load was removed. Plastic deformation resulted in residual stresses of biaxial compression under the indenter and tensile hoop stress outside the indented region.

Disk specimens were fabricated for this study from $12.7 \mathrm{~mm}$ thick plate of aluminum alloy 2024-T351. The T351 temper indicates solution heat treat followed by rapid quenching and then plastic stretching of $1-3 \%$ in the rolling direction for stress relief. Slitting method [34] tests on the as-received plate showed that the residual stresses had magnitudes of $10 \mathrm{MPa}$ or less in both in-plane directions. The average grain width in the plate was $30 \mu \mathrm{m}$ in the short-transverse (plate thickness) direction, $340 \mu \mathrm{m}$ in the rolling direction, and $160 \mu \mathrm{m}$ in the long transverse direction. Compression testing revealed no elastic anisotropy but significant plastic anisotropy, with the yield strength in stretching and through-thickness directions about $360 \mathrm{MPa}$ compared to $320 \mathrm{MPa}$ in the in-plane direction transverse to stretching.

The disks were indented quasi-statically with a peak load of $99.6 \mathrm{kN}$ [28]. Unlike the previous case of a stainless steel with isotropic plasticity [31], an accurate FE model of the indentation process in 2024-T351 has not yet been constructed because plastic anisotropy cannot be modeled at the same time as combined cyclic hardening in common commercial software.

Figure 4 illustrates the test configuration. The origin of the cylindrical coordinate system was the center of the disk. 


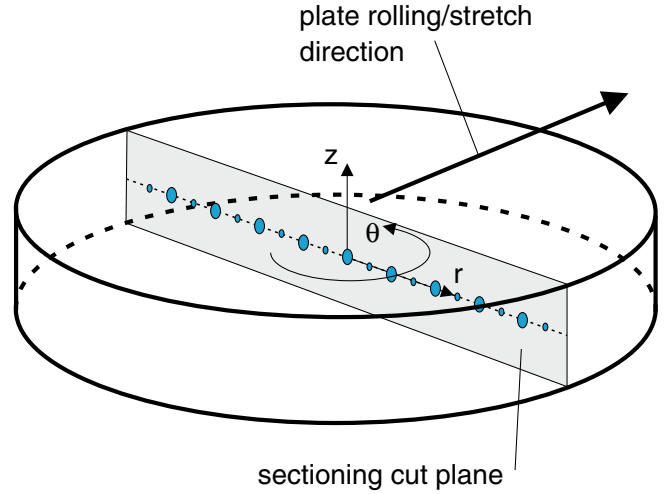

Fig. 4 Contour method sectioning plane orientation in aluminum disk. All residual stress measurements were performed on this plane. Locations of holes that were drilled later are shown on mid-thickness of cut plane

A diametrical plane oriented approximately normal to the rolling and plastic stretch direction of the plate was the plane on which all measurements were taken and on which the disk was sectioned. For this cut plane and cylindrical coordinates, equation (2) becomes

$$
\begin{aligned}
& \sigma_{\theta}^{A}(\theta=0, r, z)=\sigma_{\theta}^{C}(0, r, z) \\
& \sigma_{z}^{A}(0, r, z)=\sigma_{z}^{B}(0, r, z)+\sigma_{z}^{C}(0, r, z) \\
& \sigma_{r}^{A}(0, r, z)=\sigma_{r}^{B}(0, r, z)+\sigma_{r}^{C}(0, r, z) \\
& \tau_{r z}^{A}(0, r, z)=\tau_{r z}^{B}(0, r, z)+\tau_{r z}^{C}(0, r, z) .
\end{aligned}
$$

where for convenience in the equation, the entire cut plane is defined by $\theta=0$. Although the superposition method could be applied to any location on the sectioning plane, time considerations limited the surface stress measurements to a linear scan of $\sigma_{r}$ and $\sigma_{z}$ along the mid-thickness $(z=0)$. The nearly axisymmetric nature of the stresses meant that the shear stresses released on the cut plane, $\tau_{r \theta}$, and $\tau_{z \theta}$, could reasonably be assumed small. Therefore, the superposition results were considered separately on each half of the disk rather than averaged.

\section{Sectioning and Contour Method}

For the sectioning operation, established contour method procedure was used to map the hoop stresses. The cut was made using wire electric discharge machining (EDM) using a 100- $\mu \mathrm{m}$-diameter brass wire. Skim-cut settings were used to minimize introduced stresses [30]. The cutting direction relative to the coordinates used to plot results was such that the cut started at the $r=+30 \mathrm{~mm}$ edge and completed at $r=-30 \mathrm{~mm}$. A test cut in the same material indicated that the cut width was $130 \mu \mathrm{m}$. The specimen was clamped on both sides of the cut during cutting to minimize deformation as the stresses relaxed. After cutting, the specimen was removed from the fixture and the contours of the cut surfaces were measured on a $0.1-\mathrm{mm} \times 0.04-\mathrm{mm}$ grid using a scanner with a confocal laser ranging probe [35]. The cutting and contouring were repeated on an unindented disk as a control to confirm the cut flatness in stress-free material.

The raw contour data was processed to calculate residual stresses using established procedure [11, 31, 35]. The data from each of the two halves were interpolated onto a rectangular grid and then the two were averaged. The averaged data was smoothed using quadratic splines with knot spacing of $3.33 \mathrm{~mm}$, giving about $1-\mathrm{mm}$ spatial resolution for the surface shape. A 3D mesh of half of the disk was constructed using 51,920 linear hexahedral 8-node elements with reduced integration (C3D8R). To compute residual stresses from the smoothed data, the initially flat surface in the FE model was elastically deformed using $\mathrm{x}$-direction displacement boundary conditions into the opposite shape of the measured contour with the ABAQUS code [36]. From the results of the mechanical testing of the base material, the elastic modulus was $73.2 \mathrm{GPa}$ and a Poisson's ratio was 0.33 . From this single FE calculation, all of the $\sigma^{C}$ stresses from equation (4) were determined on the cut surface. The random uncertainties in the contour method residual stresses were estimated pointwise [35] with average one standard deviation uncertainties of about $\pm 12 \mathrm{MPa}$ for the hoop stress and about half that for the in-plane stresses. Systematic errors of similar magnitude are possible [27].

\section{Surface Preparation}

The cut surfaces were prepared for stress measurement. First, the EDM-affected layer was removed by wet polishing with 600 grit $\mathrm{SiC}$ paper. Wet polishing with 800 grit $\mathrm{SiC}$ paper was then used to remove scratches from the first polish. Finally, electropolishing for 6 min with $10 \mathrm{~V}$ applied in a $65^{\circ} \mathrm{C}$ mixed phosphoric/sulphuric acid bath was used to remove approximately $115 \mu \mathrm{m}$ from each surface.

\section{X-ray Surface Stress Measurement}

On one half of the disk, the radial and axial stresses along the mid thickness $(z=0)$ of the cut surface were measured using a local strain technique on a Philips X'Pert X-ray diffractometer using $\mathrm{Cu} \mathrm{K} \alpha$ radiation [37-40]. Measurements were taken approximately every $5 \mathrm{~mm}$ from $r=0$ to $r= \pm 25 \mathrm{~mm}$. The irradiated area was $3 \mathrm{~mm}^{2}$. The position of the peak arising from diffraction from the $\mathrm{Al}\{422\}$ planes was measured $\left(136^{\circ}<2 \theta<139^{\circ}\right)$. Sixteen scans were performed for each stress measurement using different $\psi$ values within the range $0 \leq \psi \leq 60^{\circ}$ ( $\psi$-angle between the surface normal and the bisector of source and diffracted $\mathrm{X}$-ray beam). The resulting spectra were analyzed using Philips X'pert Stress Software (version 1.0a) with peak 
locations determined with a Pearson VII fitting technique. In all cases these 16 scans were used to calculate the curve fit in the $d$ (lattice spacing) versus $\operatorname{Sin}^{2} \psi$ plots. The X-ray elastic constant, $(1 / 2) \mathrm{S}_{2}$ was $19.07\left(\mathrm{TPa}^{-1}\right)$ taken from literature for the $\{422\}$ planes [41]. Errors displayed on the figures were calculated from the standard error of the best straight line fit.

\section{Hole Drilling Surface Stress Measurement}

On the other half of the disk, the radial and axial stresses along the mid thickness $(z=0)$ of the cut surface were measured using Electronic Speckle Pattern Interferometry (ESPI) hole drilling [42, 43]. First, nine holes were drilled every $6 \mathrm{~mm}(r=0, \pm 6, \pm 12, \pm 18, \pm 24 \mathrm{~mm}$, see Fig. 4) using a 1.59-mm diameter drill with 15,000 RPM rotational speed and a feed rate of $0.05 \mathrm{~mm} / \mathrm{s}$. Next, 10 holes were drilled between and outside the previous holes ( $r= \pm 3, \pm 9, \pm 15, \pm 21, \pm 27 \mathrm{~mm}$ ) using a 0.79 -mm diameter drill with a 10,000 RPM rotational speed. Rotational speeds and feed rates were selected by checking for drilling-induced stress effects in stress-free material. All holes were drilled in four $0.1 \mathrm{~mm}$ depth increments with ESPI measurements taken between each increment. Based on the analytic solution for the stress concentration around a hole, the neighboring holes were expected to have less than $2 \%$ effect on measured stresses for the first 9 holes and $5 \%$ for the last 10 holes. Stresses were calculated using the full displacement field given by the ESPI data [44]. The stress from the shallowest $(0.1 \mathrm{~mm})$ depth increment was taken as the surface stress for superposition. One standard deviation random uncertainties for these measurements were estimated at $\pm 10-15 \mathrm{MPa}$ with possible systematic errors of similar magnitude $[45,46]$.

\section{Neutron Diffraction Measurements}

Neutron diffraction (ND) measurements were made using the SMARTS instrument [47] at the Los Alamos Neutron Science Center (LANSCE). Because neutron measurements were performed after the other measurements, a separate disk specimen that was nominally identical to the sectioned one was used. Measurement were taken along the midthickness line, $z=0$. Measurement locations were separated $1.7 \mathrm{~mm}$ near the center of the disk and by $3.4 \mathrm{~mm}$ beyond a radius of $12 \mathrm{~mm}$. The gauge volume was confined to $2 \times 2 \times$ $2 \mathrm{~mm}^{3}$ using incident slits and radial collimators. The count time for each measurement was about $30 \mathrm{~min}$. Because of the small gauge volume and the relatively weak scattering from aluminum, the diffraction patterns were only analyzable using full-pattern Rietveld analysis [48, 49]. Rietveld analysis leads to the determination of an average lattice parameter, $a_{i}$, based upon all available peaks, where $i$ indicates the direction relative to the specimen; $i=r, \theta, z$ for the radial, hoop and axial directions, respectively. The typical error-bar on the measured lattice parameters, taken as the estimated standard deviation from the Rietveld refinement, is about $75-150 \mu \varepsilon$ (microstrain, $\times 10^{-6}$ or equivalent to parts per million), depending on the position within the sample.

One advantage of the Rietveld analysis is that the lattice parameters, and thus lattice strains, determined from the refinements are average values representative of the bulk, and hence the residual stress components can be evaluated directly using Hooke's law employing the regular bulk elastic constants [50]. The lattice strains were calculated from equation (5):

$\varepsilon_{i}=\frac{a_{i}}{a_{i}^{0}}-1, i=r, \theta, z$.

Stress-free reference lattice parameters, $a_{i}{ }^{0}$, were measured on small cubes cut from the same plate as the indented disk. Based on additional neutron measurements through the thickness of the low-stress stretched plate compared with the precise slitting measurements, one standard deviation random uncertainties were estimated at $\pm 15 \mathrm{MPa}$. $15 \mathrm{MPa}$ was about $50 \%$ larger than the estimate based on peak fitting uncertainties alone, and that was probably the result of other error sources that were not accounted for. Because of the $a_{i}{ }^{0}$ measurements, a systematic shift of similar magnitude to the random uncertainties was possible.

\section{Results}

Figure 5 shows the neutron diffraction residual stress results on the mid-thickness of the plane indicated in Fig. 4.

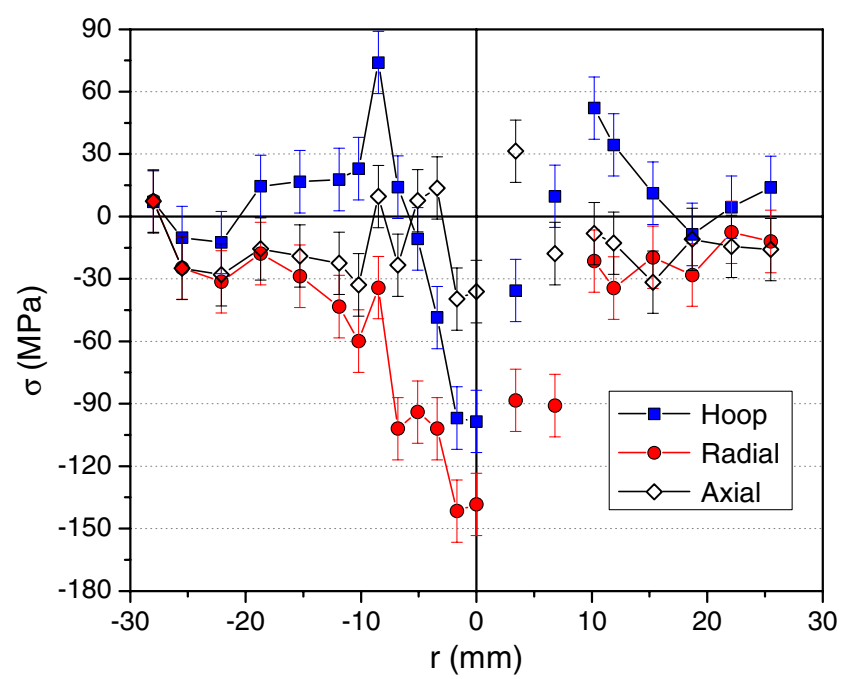

Fig. 5 Neutron diffraction stresses measured on the disk at midthickness 
Because of time limitations, not all planned measurements for positive radii were completed. Where measurements were taken at the planned resolution with overlapping volumes, data points are connected by lines in order to guide the eye. The stresses show the expected trends of nearly biaxial compression in the indented region and tensile hoop stress outside that region. For axisymmetric stresses, the hoop and radial stress would need to be equal at $r=0$ by the equilibrium condition. However, because of the plastic anisotropy, the stresses are asymmetric and the observed difference is allowed. The difference is approximately equal to the difference between flow stresses in the two in-plane directions of the disk. The axial stresses are small magnitude because of the small disk thickness and because the plastic strains induced by indentation have less spatial variation in the axial direction.

Figure 6 shows the average of the surface contours measured on the opposing surfaces created by cutting the disk in half. The surface contour was high in the central region of the disk with a peak-to-valley magnitude of about $35 \mu \mathrm{m}$. The average surface contour measured on the unindented disk as a control was flat to within measurement resolution, confirming the quality of the EDM cut.

Figure 7 shows the 3D FE model of the half-disk after it has been elastically deformed on the cut surface into the opposite of the measured contour (Fig. 6). In this state, the stresses are the change stresses, $\sigma^{C}$ from equation (1). The $x$ direction stresses, which on the cut plane are the hoop stresses, are colored in Fig. 7. Note that the stresses decay to near zero away from the cut surface.

Figure 8 shows, on the sectioning plane of Fig. 4, the stresses returned by the contour method data analysis of

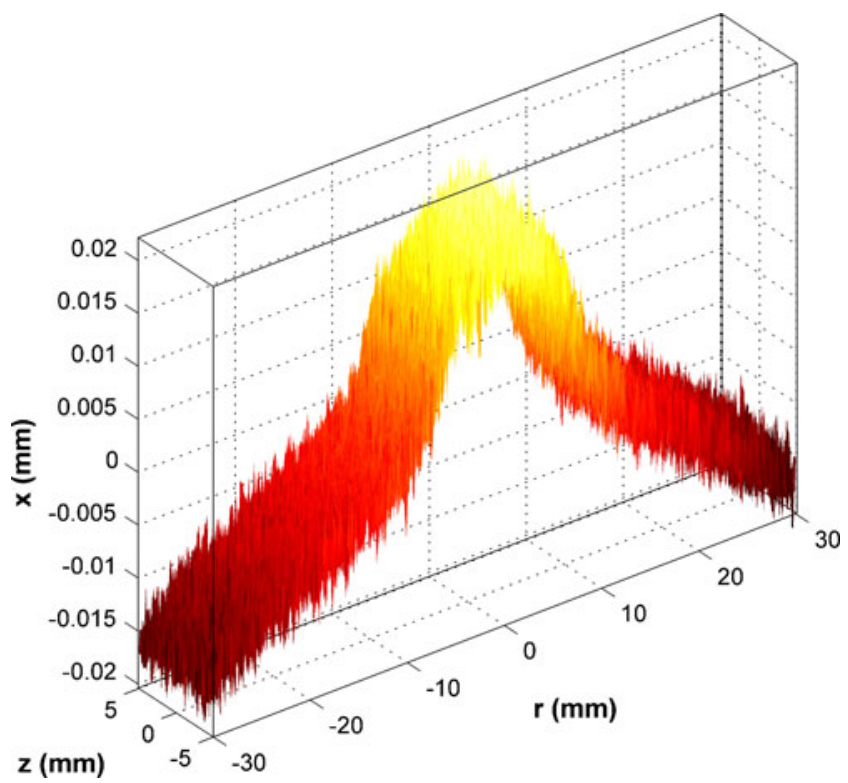

Fig. 6 Surface contour measured on cut plane (Fig. 4) of indented disk

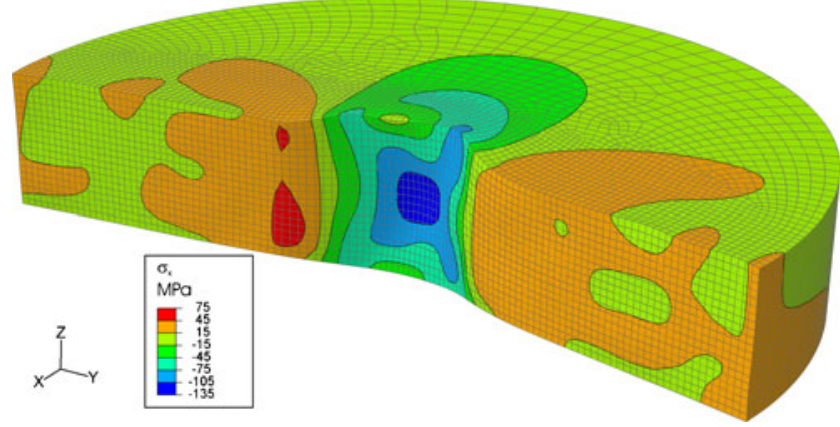

Fig. 7 3D finite element model used to calculate stresses for the contour method. X-direction boundary conditions have forced the cut surface into the opposite of the measured surface contour of Fig. 6. Displacements are exaggerated by a factor of 200

Fig. 7. These are the $\sigma^{C}$ stresses from equation (4), and without superposition only the hoop stresses are equal to the original residual stresses. In Fig. 9 the hoop stresses extracted along the mid-thickness are compared directly with the neutron results. Based upon the spatial variation observed for the contour method, see Fig. 8, the spatial averaging of the neutron data over the 2-mm cube sampling volumes does not significantly affect the comparison. Agreement is generally good, with most of the points being within the one standard deviation uncertainties. The peak stress in the contour results is $1 \mathrm{~mm}$ off center. Because of the precision fabrication and indentation, the real stresses are expected to be centered to within $0.1 \mathrm{~mm}$ of the disk center. The error is postulated to be caused by deviations from the assumption that the cut width is constant relative to the undeformed part. A more robust clamping arrangement, e.g., [51], should reduce such errors, or it could be corrected for [27]. Metallographic examination revealed that the rolling direction was about $20^{\circ}$ away from the normal to the cut plane for the specimen measured by the contour method. The neutron specimen was aligned correctly as shown in Fig. 4. An analysis based on the plastic anisotropy measured in compression testing estimated that the residual stress difference between these two orientations to be $4 \mathrm{MPa}$ or less, which is insignificant compared to the uncertainties.

Figures 8 and 9 are the results from a traditional contour method analysis and where the measurement would traditionally finish. To determine the original radial and axial stresses with the new procedure proposed in this paper, measured surface stresses were superimposed pointwise using equation (4) with contour results extracted from Fig. 8 at the appropriate point locations. Uncertainties from individual measurements were combined in quadrature to estimate the uncertainties in the sum. Figure 10 shows the radial stress superposition, with X-ray results on one half of the disk and hole drilling on the other half. The shapes of the stress distributions are quite different for the remaining 
(a) Radial stress

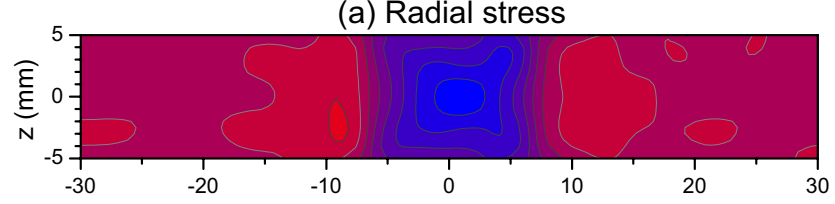

(b) Axial stress

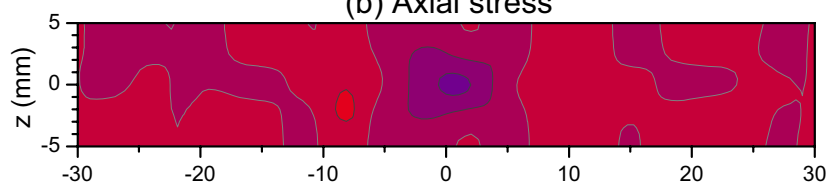

(c) Hoop stress
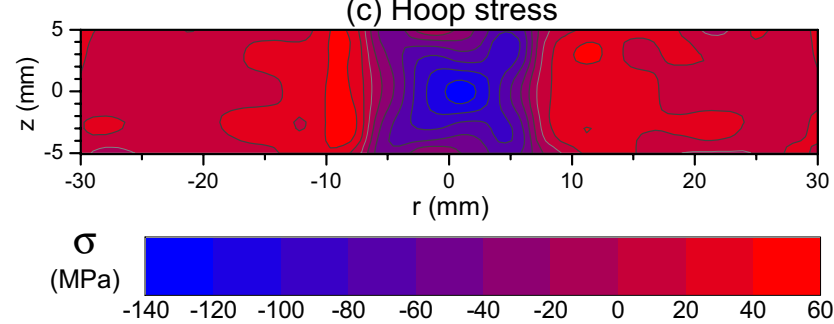

Fig. 8 Maps of the $\sigma^{C}$ stress [equation (4)] determined by the finite element model analysis of the contour method data. The lighter colored contour line is zero stress

stresses, $\sigma^{B}$, and the change stress, $\sigma^{C}$, indicating the importance of both of the contributions to superposition. Figure 11 shows the axial stress superposition with X-ray results on one half of the disk and hole drilling on the other half. The axial stresses are generally of small magnitude.

Figure 12(a) compares the radial stresses reconstructed using superposition with the neutron results from the nominally identical second disk. The reconstructed radial stresses agree within uncertainty bars with the neutron results at about $85-90 \%$ of the locations, which is better than expected for one standard deviation uncertainties. The

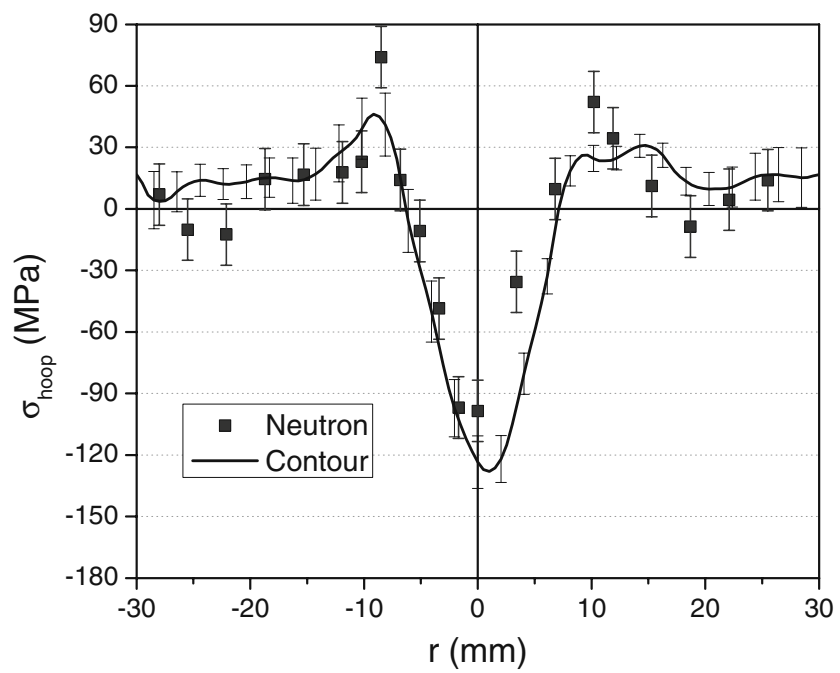

Fig. 9 The contour method measurements of hoop stress (from Fig. 8) along the disk mid-thickness ( $z=0$ Fig. 4) compared with neutron diffraction results from an identical disk (a)

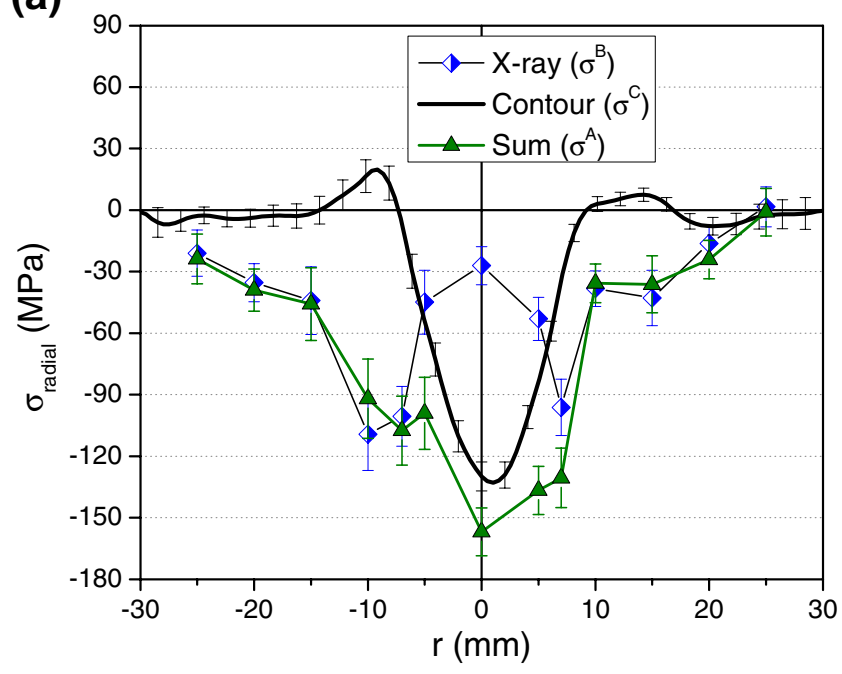

(b)

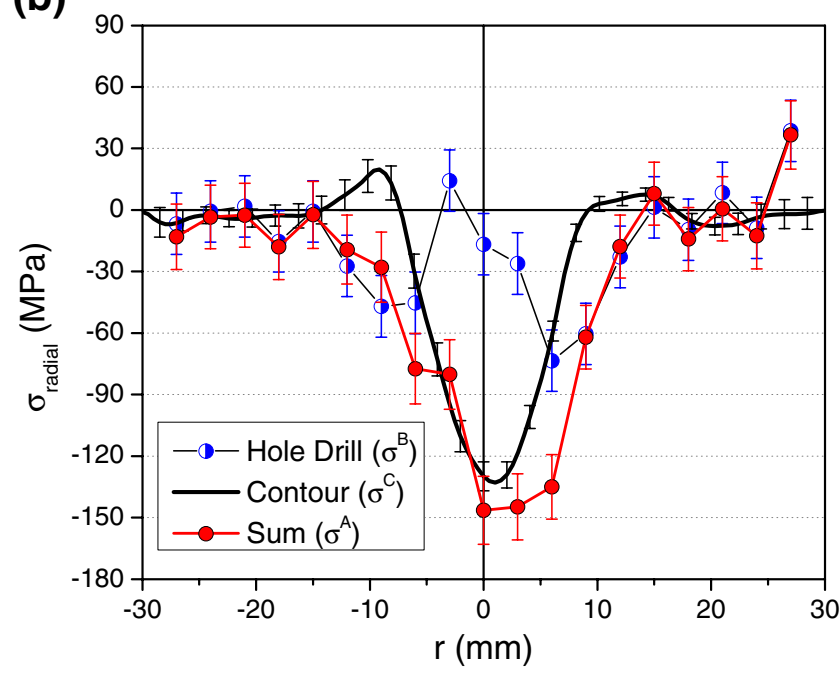

Fig. 10 Radial stress superposition from equation (4), a X-ray and b hole drilling on opposite halves

$\mathrm{X}$-ray and hole drilling reconstructions agree better with each other and with neutron results for positive radii than for negative. The $+r /-r$ asymmetry in the contour results, see Fig. 10, which peaks at $30 \mathrm{MPa}$ at $r= \pm 6 \mathrm{~mm}$ and is under $10 \mathrm{MPa}$ after $r= \pm 11 \mathrm{~mm}$, explains only part of the difference. Most of the difference is in the surface stress measurements, especially an apparent negative bias in the $\mathrm{X}$-ray results for negative radii.

The small magnitude axial stresses, compared in Fig. 12(b), are a good test of superposition because measuring low stress levels accentuates errors. The hole drilling reconstructed stresses agree with the neutron results within uncertainty bars about $85 \%$ of the time. The X-ray reconstructed stresses agree with neutron about $74 \%$ of the time, still within expectations for one standard deviation uncertainties, but seem to again have a noticeable negative bias for negative radii. The neutron results 
(a)

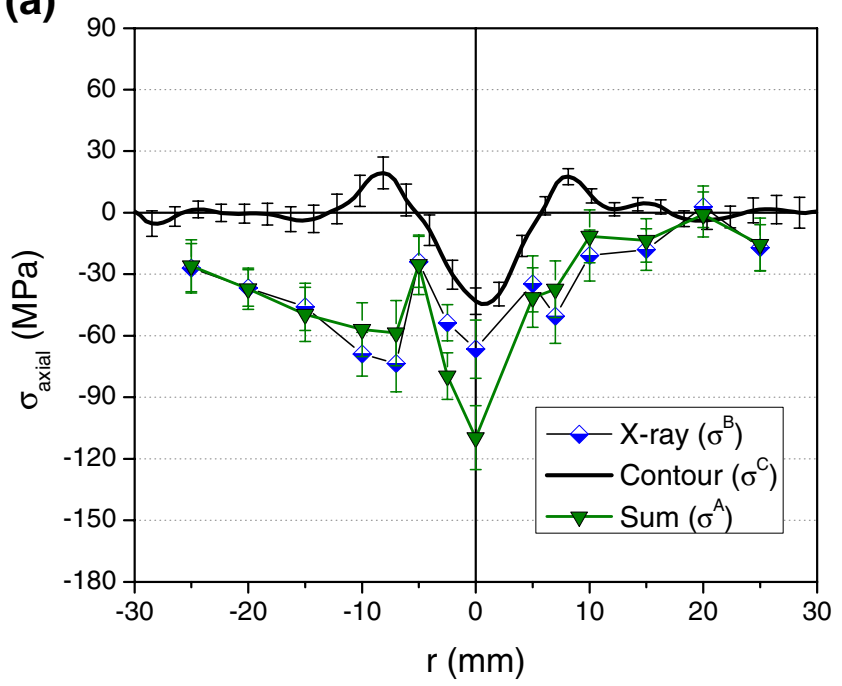

(b)

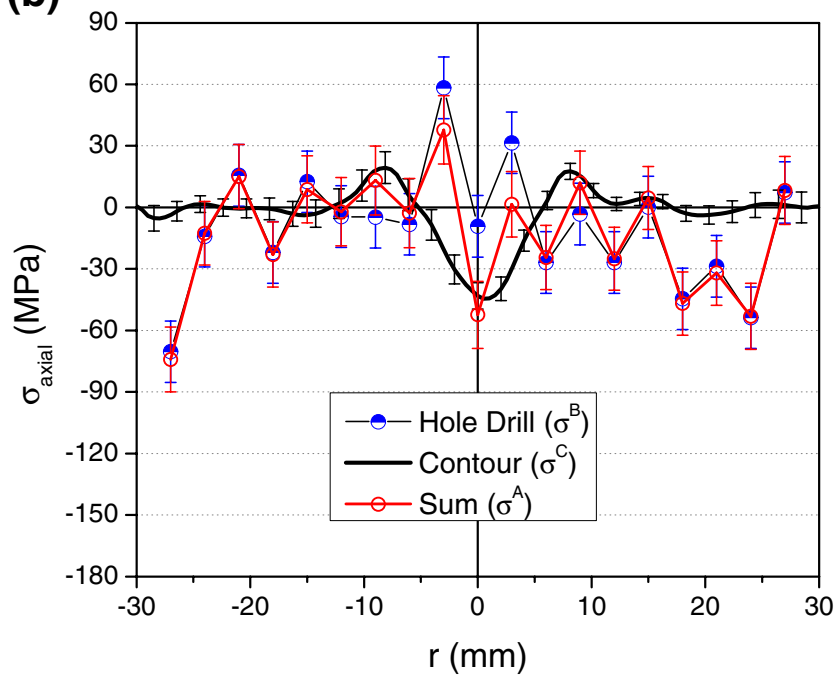

Fig. 11 Axial stress superposition from equation (4): (a) X-ray and (b) hole drilling on opposite halves of the disk

also seem to have a slight negative bias, indicating a possible offset in the measured $a^{0}$ values. The axial stresses show a subtle " $\mathrm{M}$ " shape near the center of the disk with a compressive peak at $r=0$. This feature is seen in preliminary FE simulations where the predicted axial stress is about $-70 \mathrm{MPa}$ at $r=0$ and $+30 \mathrm{MPa}$ at $r= \pm 4 \mathrm{~mm}$. The axial stress is expected to be within $10 \mathrm{MPa}$ of zero for $|\mathrm{r}|>10 \mathrm{~mm}$, which is best matched by the hole drilling reconstructed stresses.

\section{Discussion}

The experimental implementation of the superposition theory was validated by the independent neutron diffraction measurements. The agreement is impressive considering the vastly different assumptions and biases inherent to the four measurement methods included in the results: the contour method, hole drilling, X-ray diffraction, and neutron diffraction.

Because $\sigma^{B}$ stresses were measured on the cut surface, the validation involved probably the most challenging implementation of the theory, so better results can be expected when post sectioning stresses are measured subsurface. As seen in Fig. 2, the change stresses, $\sigma^{C}$, are largest on the cut surface and decay farther away in accord with St. Venant's principle. Since the change stress is largest on the cut surface, the correction to subsequently measured stresses is at its largest and the sum is then more subject to errors. Furthermore, surface stresses can be especially challenging to measure accurately because of the effects of surface preparation and irregularities, surface corrosion, etc.

(a)

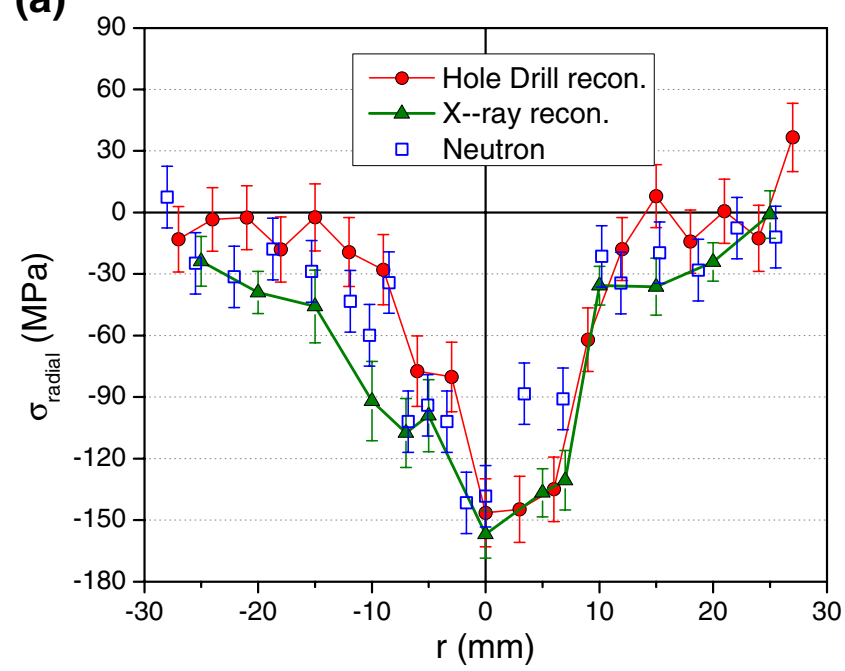

(b)

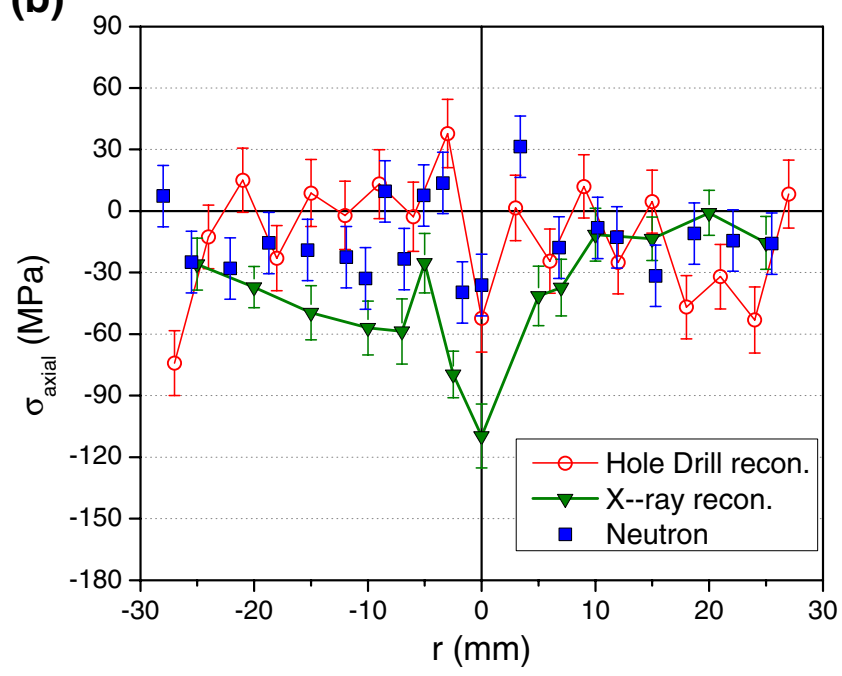

Fig. 12 Reconstructed stresses from Figs. 10 and 11 compared with neutron, (a) radial and (b) axial 
The surface-superposition contour method demonstrated in this paper extends the traditional contour method to the measurement of multiple stress components. Other techniques to measure multiple components with the contour method have different abilities and limitations. The "multiaxial" contour method gives all the stress components by making additional cuts at $45^{\circ}$ from the first cut, requiring an extruded cross section, and limiting the application to a continually processed part [52, 53]. The multiple cuts method makes multiple orthogonal cuts and reconstructs the original residual stresses using multiple superpositions [29]. Therefore, it gives the multiple stress components on different planes. The surface superposition contour method presented in this paper gives multiple stress components on a single plane using a single cut. However, it requires a surface stress measurement technique in addition to the contour method.

Under a certain condition, the surface-superposition contour method special case of the theory may be sensitive to plasticity errors. Solving equation (2) for the remaining surface stresses gives:

$\sigma_{y}^{B}(0, y, z)=\sigma_{y}^{A}(0, y, z)-\sigma_{y}^{C}(0, y, z)$

$\sigma_{z}^{B}(0, y, z)=\sigma_{z}^{A}(0, y, z)-\sigma_{z}^{C}(0, y, z)$.

Examination of Fig. 8 illustrates that the change stresses, $\sigma^{C}$, tend to be the same sign in all three directions, and, by equation (2), $\sigma_{x}^{C}(0, y, z)=\sigma_{x}^{A}(0, y, z)$. Therefore, if either of the original in-plane stresses $\sigma_{y}^{A}(0, y, z)$ or $\sigma_{z}^{A}(0, y, z)$ were of opposite sign to $\sigma_{x}^{A}(0, y, z)$, the remaining surface stresses would increase in magnitude according to equation (6). At high enough magnitude, these stresses could exceed the yield strength and potentially invalidate the assumption of elastic superposition. However, this yielding issue is expected to occur infrequently because residual stresses where the stress components have opposite signs are relatively uncommon. Also, the change stress magnitude decays rapidly away from the surface, so this should have less effect on subsurface measurement superposition. This localized surface plasticity in the transverse direction also does not have a large effect on the measured surface contour. Therefore, the traditional contour method has shown itself to be fairly insensitive to plasticity errors, as shown by the agreement with neutron diffraction results in welds where the stresses tend to be high [11, 35, 54-57].

Applying the superposition approach to axial stresses in a girth weld, the example mentioned in the Introduction, would work best with an additional step, as illustrated in Fig. 13. Because the hoop residual stresses across the ring thickness can have a net bending moment, one should first split the ring by cutting through the thickness [Michael R. Hill, U.C. Davis, personal communication]. Measuring the amount the ring opens or closes then allows a simple

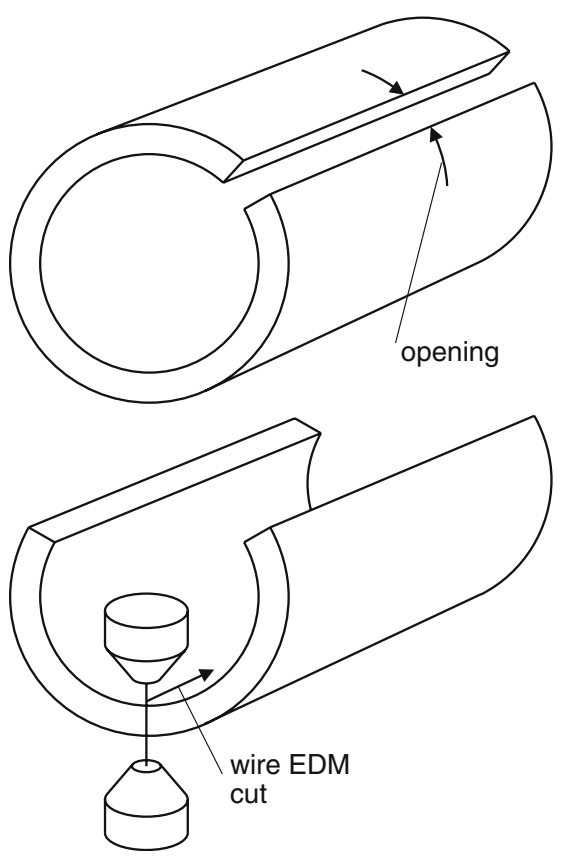

Fig. 13 Proposed multi-cut process for measuring cylinders. The amount of opening, measured after the first cut, is used to determine the released bending moment prior to the final EDM cut used for the contour method

analytic or FE calculation of the moment stresses that have been relieved [26]. Another cut, which has negligible effect, is used to remove something like a $120^{\circ}$ section of the cylinder to provide access for the final cut. That final cut, halving the remaining $240^{\circ}$ section of the cylinder, is used to measure the hoop stresses with the contour method. Now the cross section is exposed for measurement of surface axial and radial stresses. The two-step procedure has already been validated for measuring the hoop stresses but not the axial [58].

\section{Conclusions}

1. A theory was presented for using superposition to determine internal residual stresses by sectioning with the contour method and then measuring remaining stresses with other methods. This theory can allow measurements in parts where the internal stresses were previously difficult to access. This approach opens up possibilities to combine the advantages of different techniques to obtain unprecedented measurements and understanding.

2. A special case of the theory was presented, the surface superposition contour method, for measuring triaxial stress states on the plane of the cut. It was implemented on a test specimen using X-ray diffraction and hole drilling for surface stress measurement and then 
experimentally validated by comparing with neutron diffraction measurements.

3. The surface superposition contour method may be more difficult to implement accurately than subsurface measurements. Care must be taken that the layer affected by the cutting process is removed prior to surface stress measurement. Furthermore, under certain described conditions, surface stresses are more likely to be affected by local plasticity.

Acknowledgements MBP would like to thank Iain Finnie of U.C. Berkeley for two decades of mentorship and friendship. Professor Finnie passed away in December 2009. Some of this work was performed at Los Alamos National Laboratory, operated by the Los Alamos National Security, LLC for the National Nuclear Security Administration of the U.S. Department of Energy under contract DEAC52-06NA25396. This work has benefited from the use of the Lujan Neutron Scattering Center at LANSCE, funded by the Department of Energy's Office of Basic Energy Sciences. Dr. Pagliaro's work was sponsored by a fellowship from the Università degli Studi di Palermo

Open Access This article is distributed under the terms of the Creative Commons Attribution Noncommercial License which permits any noncommercial use, distribution, and reproduction in any medium, provided the original author(s) and source are credited.

\section{References}

1. Majumdar S (1999) Failure and leakage through circumferential cracks in steam generator tubing during accident conditions. Int $\mathrm{J}$ Press Vessels Piping 76:839-847

2. Wang X, Reinhardt W (2003) On the assessment of through-wall circumferential cracks in steam generator tubes with tube supports. J Press Vessel Technol 125:85-90

3. Bush SH (1992) Failure mechanisms in nuclear power plant piping systems. J Press Vessel Technol 114:389-395

4. Dong P, Brust FW (2000) Welding residual stresses and effects on fracture in pressure vessel and piping components: a millennium review and beyond. J Press Vessel Technol 122:329-338

5. Bouchard PJ (2007) Validated residual stress profiles for fracture assessments of stainless steel pipe girth welds. Int J Press Vessels Piping 84:195-222

6. Withers PJ, Preuss M, Steuwer A, Pang JWL (2007) Methods for obtaining the strain-free lattice parameter when using diffraction to determine residual stress. J Appl Crystallogr 40:891-904

7. Krawitz A (1994) Use of position-dependent stress-free standards for diffraction stress measurements. Mater Sci Eng A 185:123-130

8. Holden TM, Suzuki H, Carr DG, Ripley MI, Clausen B (2006) Stress measurements in welds: problem areas. Mater Sci Eng A 437:33-37

9. Smith DJ, Bouchard PJ, George D (2000) Measurement and prediction of residual stresses in thick-section steel welds. J Strain Anal Eng Des 35:287-305

10. Prime MB (2001) Cross-sectional mapping of residual stresses by measuring the surface contour after a cut. J Eng Mater Technol 123:162-168

11. Johnson G (2008) Residual stress measurements using the contour method. Ph.D. Dissertation, University of Manchester

12. Lillard RS, Kolman DG, Hill MA, Prime MB, Veirs DK, Worl LA, Zapp P (2008) Assessment of corrosion based failure in stainless steel containers used for the long-term storage of plutonium base salts. Corrosion 65:175-186

13. Brand PC, Prask HJ, Gnaeupel-Herold T (1997) Residual stress measurements at the NIST reactor. Physica B. 241-243:1244 1245

14. Pearce SV, Linton VM, Oliver EC (2008) Residual stress in a thick section high strength T-butt weld. Mater Sci Eng A 480:411-418

15. Sasaki T, Takahashi S, Kanematsu Y, Satoh Y, Iwafuchi K, Ishida M, Morii Y (2008) Measurement of residual stresses in rails by neutron diffraction. Wear 265:1402-1407

16. Stacey A, MacGillivary HJ, Webster GA, Webster PJ, Ziebeck KRA (1985) Measurement of residual stresses by neutron diffraction. J Strain Anal Eng Des 20:93-100

17. Stefanescu D, Browne P, Truman CE, Smith DJ (2003) Residual stress measurement within a European UIC60 rail using integrated drilling techniques. Mat Sci Forum 440-441:85-92

18. Tawfik D, Kirstein O, Mutton PJ, Wing Kong C (2006) Verification of residual stresses in flash-butt-weld rails using neutron diffraction. Physica B 385-386:894-896

19. Buttle DJ, Culham Science Centre A, Collett N, Webster PJ, Hughes DJ, Mills G (2002) A comparison of MAPS and synchrotron $\mathrm{x}$-ray methods: stresses measured in railway rail sections. Mat Sci Forum 404-407:881-886

20. de Oliveira U, Ocelik V, De Hosson JTM (2006) Residual stress analysis in co-based laser clad layers by laboratory $\mathrm{x}$-rays and synchrotron diffraction techniques. Surf Coat Technol 201:533542

21. Djapic Oosterkamp L, Webster PJ, Browne PA, Vaughan GBM, Withers PJ (2000) Residual stress field in a friction stir welded aluminium extrusion. Mat Sci Forum 347-349:678-683

22. Webster PJ, Hughes DJ, Mills G, Vaughan GBM (2002) Synchrotron x-ray measurements of residual stress in a worn railway rail. Mat Sci Forum 404-407:767-772

23. Jun TS, Hofmann F, Belnoue J, Song X, Hofmann M, Korsunsky AM (2009) Triaxial residual strains in a railway rail measured by neutron diffraction. J Strain Anal Eng Des 44:563-568

24. McDonald EJ, Hallam KR, Bell W, Flewitt PEJ (2002) Residual stresses in a multi-pass CrMoV low alloy ferritic steel repair weld. Mater Sci Eng A 325:454-464

25. Bueckner HF (1973) Field singularities and related integral representations. Mechanics of Fracture G. C. Sih, ed., 239-314

26. Schajer GS (2001) Residual stresses: measurement by destructive testing. Encyclopedia of Materials: Science and Technology, Elsevier, 8152-8158

27. Prime MB, Kastengren AL (2010) The contour method cutting assumption: error minimization and correction. Proc. SEM Conference \& Exposition on Experimental \& Applied Mechanics, Indianapolis, IN USA

28. Pagliaro P (2008) Mapping multiple residual stress components using the contour method and superposition. Ph.D. Dissertation, Universitá degli Studi di Palermo, Palermo

29. Pagliaro P, Prime MB, Swenson H, Zuccarello B (2010) Measuring multiple residual-stress components using the contour method and multiple cuts. Exp Mech 50:187-194

30. Cheng W, Finnie I, Gremaud M, Prime MB (1994) Measurement of near-surface residual-stresses using electric-discharge wire machining. J Eng Mater Technol 116:1-7

31. Pagliaro P, Prime MB, Clausen B, Lovato ML, Zuccarello B (2009) Known residual stress specimens using opposed indentation. J Eng Mater Technol 131:031002

32. Mahmoudi AH, Stefanescu D, Hossain S, Truman CE, Smith DJ, Withers PJ (2006) Measurement and prediction of the residual stress field generated by side-punching. J Eng Mater Technol 128:451-459

33. Lam Y, Lian K (1989) The effect of residual stress and its redistribution of fatigue crack growth. Theor Appl Fract Mech 12:59-66 
34. Cheng W, Finnie I (2007) Residual Stress Measurement and the Slitting Method, Springer Science+Business Media, LLC, New York, NY, USA

35. Prime MB, Sebring RJ, Edwards JM, Hughes DJ, Webster PJ (2004) Laser surface-contouring and spline data-smoothing for residual stress measurement. Exp Mech 44:176-184

36. Abaqus 6.9, ABAQUS, inc., Pawtucket, RI, USA, 2009

37. Fitzpatrick M, Fry A, Holdway P, Kandil F, Shackleton J, Suominen L (2005) NPL Good Practice Guide No. 52: Determination of Residual Stresses by X-Ray Diffraction. National Physical Laboratory, UK

38. Prevey P (1986) X-ray diffraction residual stress techniques. Metals Handbook, K. Mills, ed., ASM International, Metals Park, OH, 380-392

39. Noyan I, Cohen J (1987) Residual stress: measurement by diffraction and interpretation. Springer-Verlag, Berlin, FRG

40. Macherauch E (1966) X-ray stress analysis. Exp Mech 6:140-153

41. Hauk V, Macherauch E (1984) A useful guide for X-ray stress evaluation (XSE). Adv X-ray Anal 27:81-99

42. Steinzig M, Ponslet E (2003) Residual stress measurement using the hole drilling method and laser speckle interferometry: part I. Exp Tech 27:43-46

43. Nelson DV, McCrickerd JT (1986) Residual-stress determination through combined use of holographic interferometry and blindhole drilling. Exp Mech 26:371-378

44. Schajer GS, Steinzig M (2005) Full-field calculation of hole drilling residual stresses from electronic speckle pattern interferometry data. Exp Mech 45:526-532

45. Ponslet E, Steinzig M (2003) Residual stress measurement using the hole drilling method and laser speckle interferometry part III: analysis technique. Exp Tech 27:45-48

46. Steinzig M, Takahashi T (2003) Residual stress measurement using the hole drilling method and laser speckle interferometry part IV: measurement accuracy. Exp Tech 27:59-63

47. Bourke MAM, Dunand DC, Ustundag E (2002) SMARTS-a spectrometer for strain measurement in engineering materials. Appl Phys A Mater Sci Process 74:1707-1709
48. Rietveld HM (1969) A profile refinement method for nuclear and magnetic structures. J Appl Crystallogr 2:65-71

49. Von Dreele RB, Jorgensen JD, Windsor CG (1982) Rietveld refinement with spallation neutron powder diffraction data. J Appl Crystallogr 15:581-589

50. Daymond MR (2004) The determination of a continuum mechanics equivalent elastic strain from the analysis of multiple diffraction peaks. J Appl Physi 96:4263

51. Hacini L, Van Lê N, Bocher P (2009) Evaluation of residual stresses induced by robotized hammer peening by the contour method. Exp Mech 49:775-783

52. DeWald AT, Hill MR (2006) Multi-axial contour method for mapping residual stresses in continuously processed bodies. Exp Mech 46:473-490

53. Kartal ME, Liljedahl CDM, Gungor S, Edwards L, Fitzpatrick ME (2008) Determination of the profile of the complete residual stress tensor in a VPPA weld using the multi-axial contour method. Acta Mat 56:4417-4428

54. Zhang Y, Ganguly S, Edwards L, Fitzpatrick ME (2004) Crosssectional mapping of residual stresses in a VPPA weld using the contour method. Acta Mat 52:5225-5232

55. Kartal M, Turski M, Johnson G, Fitzpatrick ME, Gungor S, Withers PJ, Edwards L (2006) Residual stress measurements in single and multi-pass groove weld specimens using neutron diffraction and the contour method. Mat Sci Forum 524525:671-676

56. Withers PJ, Turski M, Edwards L, Bouchard PJ, Buttle DJ (2008) Recent advances in residual stress measurement. Int J Press Vessels Piping 85:118-127

57. Smith MC, Smith AC (2009) Net bead-on-plate round Robin: comparison of residual stress predictions and measurements. Int $\mathrm{J}$ Press Vessels Piping 86:79-95

58. Brown DW, Holden TM, Clausen B, Prime MB, Sisneros TA, Swenson H, Vaja J (2010) Critical comparison of two independent measurements of residual stress in an electron-beam welded uranium cylinder: neutron diffraction and the contour method. Acta Mat. doi:10.1016/j.actamat.2010.09.022 